\title{
Implementation of Network Motion System using RTEX Network for XY Gantry
}

Byounghee Kim*, Sinwoo Song ${ }^{\$}$, Euicheong Hwang ${ }^{\$}$, Kyoungdyuk Rho ${ }^{\$}$, Jonghyo Lee ${ }^{\#}$

*\$\$Artillery System Team, Hanwha Land System R\&D Center, Korea

\#Agency for Defense Development, Korea

(Received 15 August 2018; accepted 20 February 2019)

https://doi.org/10.36224/ijes.120101

\begin{abstract}
Recently, the weapon systems are more and more complex and the demands for handling and maintaining these complex systems effectively are increased. For this reason, industrial control network had been developed for many years and the needs for the development of a totally networked automation environment are raised. To meet these requirements, networked motion control systems tend more and more to replace the traditional solutions. In this paper, the XY platform motion control system employing RTEX (Real Time Express) network which is one of the Ethernet based vender-oriented industrial networks is tackled. The network motion controller which generates motion profile and outputs the pulse train is implemented and is applied to XY platform. As results, it is shown that the proposed network motion controller works properly to control the XY platform and monitors the system easily through network.
\end{abstract}

Keywords: Network motion, motion controller, motion profile, XY platform control

\section{Introduction}

In recent years, systems for manufacturing and production are becoming more and more complex. More and more motorized devices are being used in manufacturing or production lines to attain the complex motions that are required for today's sophisticated manufacturing techniques. To control the devices in these lines, analog control has been used, but the vast amount of wiring required for analog control makes it difficult to connect a large number of devices. The functions that analog control can offer are too limited to satisfy the demand for higher levels of control. Now, control using digital communications is increasing in demand and taking the place of analog control. Also, to meet the demand for increased productivity, a cycle time that is one second or even one millisecond shorter will result in a shorter control cycle between control devices. Accordingly, to get these required results, the demand for high-speed digital communications has been increasing. On the other hand, customers frequently change the models and manufacturers of their control devices to take advantage of rapidly decreasing prices and improvements in function and control performance. Most manufacturing equipment consists of two sections: a drive section and an I/O control section. Customers want to control both of these sections with the use of only one digital communications line. With regards to motion control connectivity, reducing the number of cables and increasing design flexibility is always a key. For this reason, industrial control network, so called fieldbus, had been developed for many years and the needs for the development of a totally networked automation environment are raised. Furthermore, due to the quick evolution of manufacturing processes, the demand for more flexible automation systems is on the rise. To meet these requirements, networked motion control systems tend more and more to replace the traditional solutions. The primary advantages of a networked motion control system are reduction of system wiring, easy of system diagnosis and maintenance, and high system agility, etc. Recently, because of the quick manufacturing 
processes, the Ethernet based industrial network becomes commonly used in many motion control areas.

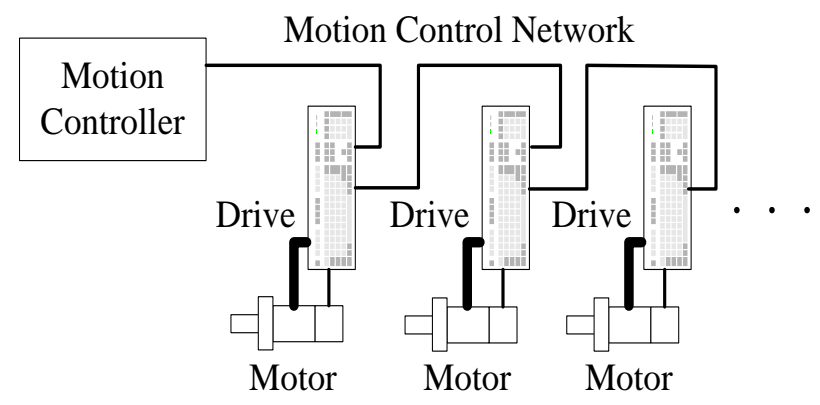

Figure 1: Network motion control system.

Figure 1 shows the networked motion control system which is composed of servo controllers with network interface, motors, a motion controller and a control network. Networked motion requires a control network that supports high-speed transmission of control command and feedback information. Recently researches about networked motion control systems have been increased with years. Lorenz presented three approaches which are synchronized master command approach, master-slave approach and relative dynamic stiffness approach 1. Fuzzy logic coupling control and neuro-controller approach is also endeavored 2,3. Niemann discussed fieldbus-based motion control strategies 4. Samaranayake presented a speed controller for distributed motion control via Ethernet 5. Hsieh was put forward a CANBUS-based motion control approach 6.

In this paper, the network motion controller is implemented using RTEX network which is the Ethernet based network and applied to shaft motor test bed to show the control performance. In section 2, the motion control networks are introduced. The implementation of the network motion controller is discussed in section 3. In section 4, the experimental results that are applied to shaft motor bed are analyzed. Finally, we make conclusions in section 5.

\section{Motion Control Networks}

Many Ethernet based control networks have been developed for use in networked motion control systems. In this paper, vender-oriented networks are introduced since the RTEX which is one of the vender-oriented networks is applied. The main reason of selection of the RTEX is that the RTEX network can control 16 axes basically and be extended up to 32 axes without increasing communication time. Another reason is the communication speed which is $100 \mathrm{Mbps}$ and application examples. Still another reason is that many industries have been used Panasonic's drive and motor for many years which means easily upgrade to network type system from non-network type system.

\section{Implementation of Network Motion Controller}

In this section, the implementation of network motion controller is introduced. Since the exclusive servo drive is used, the proposed network motion controller generates motion profile and output the position command. The proposed network motion controller transmits the generated motion profile and receives encoder feedback data through general Ethernet cable which satisfies CAT-5E. 


\subsection{Hardware Design}

The proposed network motion controller mainly consists of three parts: motion profile generation part, communication part, and bus interface part. Figure. 2 shows the proposed network motion controller block diagram.

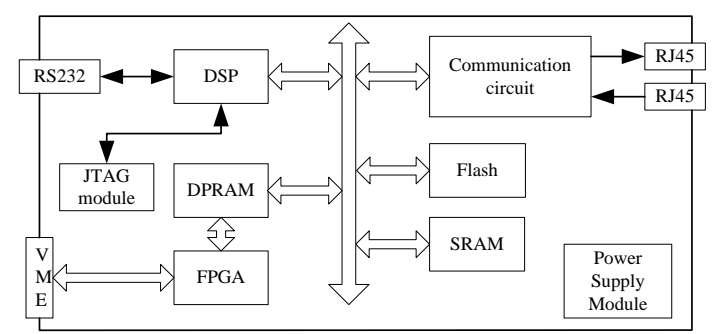

Figure 2: Proposed network motion controller

The motion command and monitoring data can transmit and receive through the RS232 port. Since the XY gantry system consists of VME bus system, FPGA plays a role of VME interface. The communication circuit consists of Panasonic communication chip and PHY chip which is commonly found in Ethernet device and connects a link layer device to a physical medium since RTEX is Ethernet based motion control network. The communication period of the proposed network motion controller is $0.5 \mathrm{~ms}$ and can control up to 32 axes by itself. As mentioned before, one advantage is that even the axis increased up to 32 , the communication period is fixed at $0.5 \mathrm{~ms}$.

\subsection{Motion Profile Planning}

Due to the global competency, there is an increasing demand for high-precision and highspeed automatic assembly equipment. An important issue for such equipment is the jerk and vibration caused by the inappropriate acceleration or deceleration motion, which results in decreased accuracy and increased settling time to the positioning table. To solve such problems and reach a high production efficiency level, the new-generation motion controller must have the capability to plan trajectory and generate motion profile in such a way that the accelerating and decelerating phases become much smoother to reduce inertia force and decrease vibration of point-to-point motion. A typical motion velocity profile can be divided into two categories: trapezoidal and s-curve profile. It is already known that the s curve velocity profile is superior to the trapezoidal velocity profile in aspect to setting time, accuracy and vibration. The s-curve profile, however, still exhibit a sudden change of jerk profile, and the finite jerk spreads out over a period of time 11 . Therefore, many researches have been developed to reduce the vibration and improve the motion performance 8 - 10. For example, Macfarlane and Croft 7 presented a method using a concatenation of fifth-order polynomial to provide a smooth trajectory for pointto-point motion with jerk limits and Meckl et al. 11 proposed an approach to optimize the selection of the ramp-up and ramp-down time for s-curve motion profiles to reduce the residual vibration. In this paper, the simple sine wave function is used to generate s-curve velocity profile which is expected a smooth jerk profile. For maintaining the optimal CPU performance and reducing calculation time, the table method is used. The velocity, acceleration, jerk, and displacement profiles which are used in the proposed motion controller are shown in Figure 3. (Only acceleration portion). 

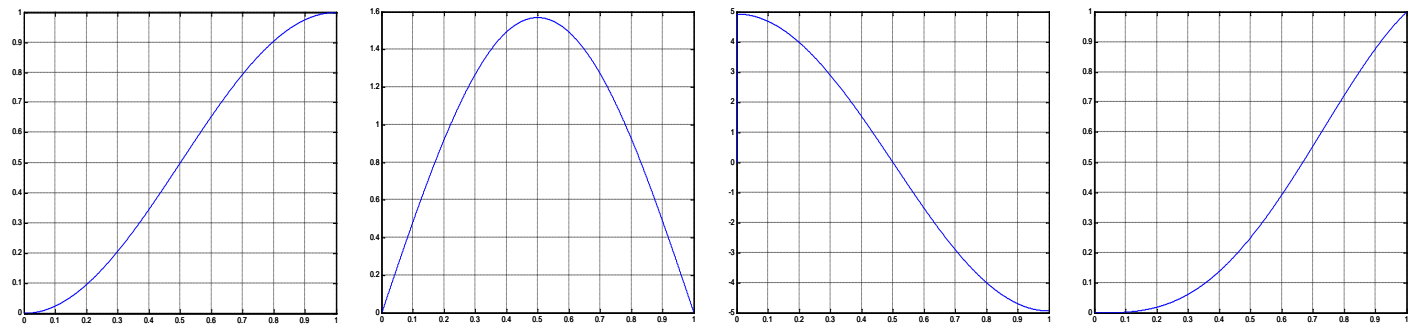

Figure 3: proposed motion profile

The profiles are generated for the arbitrary time $t$ and normalized. Since the sine wave function is applied for the velocity profile, the shape of the acceleration and jerk profile is also sine wave function which means no discontinuity and can reduce the vibration of the manufacturing equipments consequently. The motion profile can simply be asymmetry by changing acceleration and deceleration time interval.

\section{Experiment results}

\subsection{XY Gantry System}

The target XY gantry system consists of the six shaft motors. The shaft motors are directdrive linear servomotors that consist of a shaft with laminated magnets and cylindrically wound coils controlled by the flow of current. These motors are a new generation of actuators based on a very simple construction that has drives which use magnetic circuits consisting only of permanent magnets and coils, and subsequently produces a wide range of characteristics. These characteristics include precision positioning and high, low and constant speed driving, making them suitable for a highly diverse range of applications. Unlike the general rotary type motors, the shaft motor uses the linear scale as a feed back signal. The XY gantry system use the GHC shaft motor which the rate force is $190 \mathrm{~N}$ for $\mathrm{X}$ axis and $250 \mathrm{~N}$ for $\mathrm{Y}$ axis, respectively and available stroke is $2500 \mathrm{~mm}$ for $\mathrm{X}$ and $\mathrm{Y}$ axes. Since the shaft motor is applied for the XY gantry system, the linear scale is used for feedback. The Mitutoyo's ST 700 series absolute linear scale is chosen which the resolution is $0.5 \mu \mathrm{m}$. Figure. 4 shows the 3D layout of the shaft motor test bed.

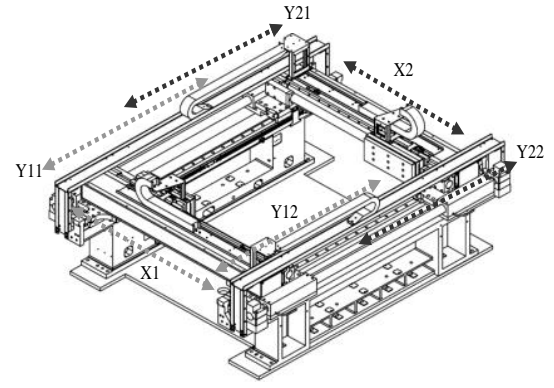

Figure 4: 3D layout of XY Gantry

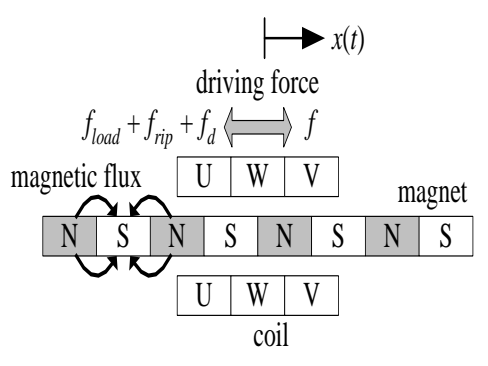

Figure 5: Structure of Shaft Motor

There are three motors for front side X1, Y11, and Y12 and other three motors for rear side X2, Y21, and Y22, respectively. Since the XY gantry consists of six shaft motors, six axes, six servo drives are connected each other with network motion controller through the network. Because the proposed network motion controller can handle up to 32 axes, as mentioned before, only one controller is enough to control the XY gantry system. The XY gantry system is a highly integrated nonlinear process that couples the dynamics of the six motors with the electro-magnetic field of the shaft which is composed laminated magnets and cylindrically wound coils. Figure 5 shows the structure of the shaft motor. 
According to the 12, the dynamics of the shaft motor can be simplified as

$f(t)=k_{f} \cdot i_{q}(t)$

$f(t)=m \dot{v}+B v+f_{\text {load }}(t)+f_{\text {rip }}(x)+f_{d}(t)$

where, $x(t)$ is the motor position, $v(t)$ and $i_{q}(t)$ are the time-varying motor terminal voltage and the equivalent armature current, respectively. $k_{f}$ is the force coefficient related with flux linkage of a shaft motor; $m$ is the mass of a carriage; $f(t)$ and $f_{\text {load }}(t)$ are the developed force and the applied load force, respectively. and $f_{\text {rip }}(x)$ denote the force ripple, $f_{d}(t)$ denotes the other force disturbances. By neglecting the nonlinear portion in Eq. (2) and combining with Eq. (1), the following linearized equation can be acquired.

$i_{q}(t)=\frac{m}{k_{f}} \dot{v}+\frac{B}{k_{f}} v$

Even the shaft motor can be modeled as Eq. (1) and Eq. (2), it is only for one axis and the movement of the combination of the six motors is highly nonlinear since each motor is coupled in the XY gantry system. Because it is quite hard to get exact mathematical model of the XY gantry system, the FFT analysis is normally used to understand the system characteristics in the field. The overall structure of the control system is shown in Figure. 6.

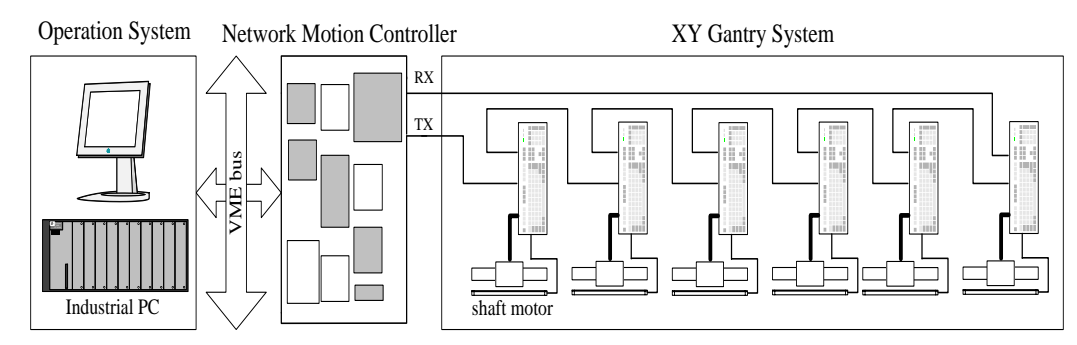

Figure 6: Overall structure of the control system.

The overall system can be divided into three parts: Operation System, Network Motion Controller, and XY Gantry System. The operation system can communicate with the network motion controller by the VME bus and the network controller is connected to the target application by RTEX network which is configured as ring topology.

\subsection{Experiment results}

Before applying the proposed network motion controller, the FFT analysis is performed to get XY gantry system characteristics. Figure 7 shows the FFT results for the front X axis and front $\mathrm{Y}$ axis, respectively.
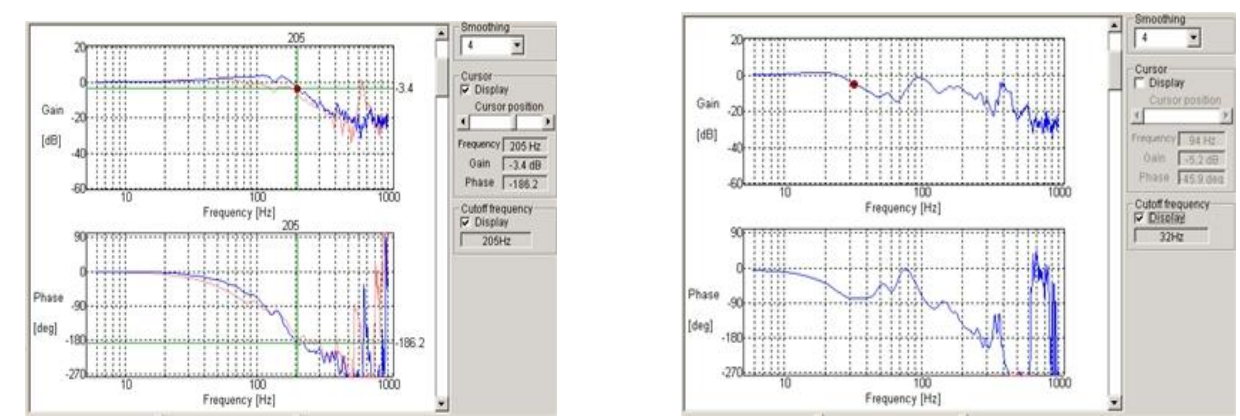

Figure 7: FFT results of front $\mathrm{X}$ and front $\mathrm{Y}$ axes 
Figure $8 \sim$ Figure 10 show the motion characteristic by applying the proposed motion profile. Three motion profiles-S curve, proposed $\mathrm{S}$ curve, polynomial $\mathrm{S}$ curve-were compared and the setting time is improved when using the proposed motion profile.

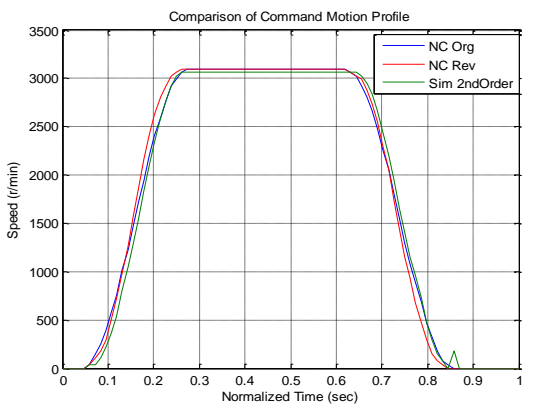

Figure 8: Comparison of Command Motion Profile.

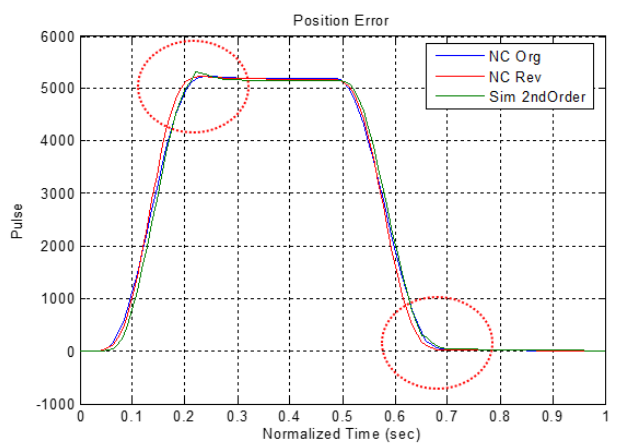

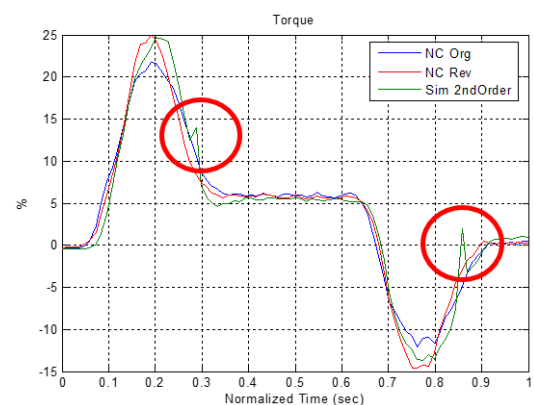

Figure 9: Comparison of Torque.

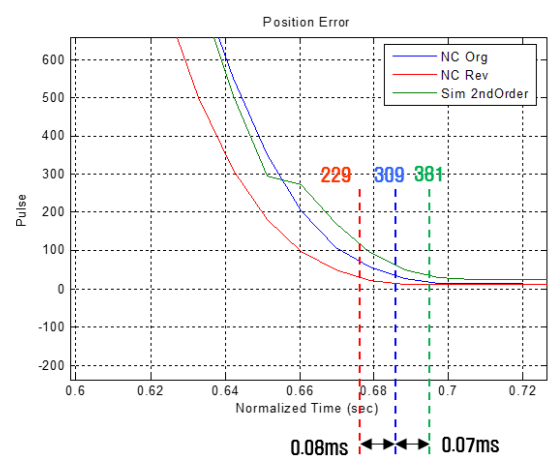

Figure 10: Comparison of Position Error.

\section{Conclusion}

In this paper, the network motion controller employing RTEX is introduced. The experiment target is XY gantry system consisting of six shaft motors and each three motors move together as front gantry and rear gantry. Since three motors are coupled, it is very difficult to get exact mathematical model. Therefore, the FFT is performed to understand system characteristics. From the results, the proposed network motion controller works properly in the XY gantry system. The setting time is under 229 and the servo parameter can be handled through the network. The proposed network motion controller can be applied to the other manufacturing equipments and the robot based applications. The future work is implementation of control scheme in the proposed network motion controller to make it general controller. By this, the network motion controller can be used more widely in motion control area.

\section{References}

1. R. D. Lorenz and P. B. Schmidt, Synchronized motion control for process automation, Proceedings of the 1989 IEEE industry applications annual meeting, 1989, $1693-1698$.

2. P. R. Moore and C. M. Chen, Fuzzy logic coupling and synchronized control of multiple independent servo-drives, Control engineering practice, 1995, 3, 1697 - 1708.

3. H. C. Lee and G. J. Jeon, A neuro-controller for synchronization of two motion axes, International journal of intelligent system1998, 39, $571-586$.

4. H. Niemann, Synchronised motion using fieldbus linked electrical drives - an architectural analysis, Proceedings of PCIM'2000 conference, 2000, 141 - 146. 
5. L. Samaranayake, S. Alahakoon, and K. Walgama, Speed controller strategies for distributed motion control via Ethernet, IEEE international symposium on intelligent control, 2003, 322 - 327.

6. C. C. Hsieh, A. P. Wang, and P. L. Hsu, CAN-based motion control design, SICE annual conference, 2003, $2504-2509$.

7. S. Macfarlane and E.A., Croft, Jerk-bounded manipulator trajectory planning: design for real-time applications, IEEE Transactions on Robotics and Automation, 2003, 19(1), 42 52.

8. P. Lambrechts, M. Boerlage, and M. Steinbuch, Trajectory planning and feedforward design for electromechanical motion systems, Control Engineering Practice, 2005, 13, 145 $-157$.

9. B.G. Dijkstra, N.J. Rambaratsingh, C.W. Scherer, O.H. Bosgra, M. Steinbuch, and S. Kerssemakers, Input design for optimal discrete time point-to-point motion of an industrial xy-positioning table, Selected Topics in Signals, Systems and Control, 2001, 914.

10. H. Z. Li, Z. Gong, W. Lin, and T. Lippa, A New motion control approach for jerk and transient vibration suppression, IEEE International Conference on Industrial Informatics, 2006, 16(18), $676-681$.

11. P. H. Meckl, P. B. Arestides, and M. C. Woods, Optimizebd s-curve motion profiles for minimum residual vibration, Proceedings of the American Control Conference, Philadelphia, 1998, $2627-2631$.

12. K. K. Tan, Precision motion control with disturbance observer for pulse widthmodulated-driven permanent- magnet linear motors, IEEE Trans. on Magnetics, 2003, 3, 1813-1818. 